Enferm Bras 2019;18(2):193-200

https://doi.org/10.33233/eb.v18i2.2077

\title{
ARTIGO ORIGINAL \\ Contribuições da integração ensino-serviço-comunidade para a formação e transformação de práticas na saúde do idoso
}

lara Sescon Nogueira, M.Sc. *, Rayane Nascimbeni Maldonado**, Célia Maria Gomes Labegalini, M.Sc. ${ }^{* *}$, André Estevam Jaques, D.Sc. ${ }^{* * *}$, Ligia Carreira, D.Sc.. ${ }^{* * * *}$, Vanessa Denardi Antoniassi Baldissera, D.Sc.*****

*Enfermeira, Doutoranda em Enfermagem do Programa de Pós-graduação em Enfermagem da Universidade Estadual de Maringá, **Enfermeira, Especialista em Enfermagem Perioperatória, Mestranda em Enfermagem da Universidade Estadual de Londrina, ${ }^{* \star *}$ Enfermeira, Doutoranda em Enfermagem do Programa de Pós-graduação em Enfermagem da Universidade Estadual de Maringá, ${ }^{* * * * E n f e r m e i r o, ~ d o c e n t e ~ d o ~ D e p a r t a m e n t o ~ d e ~ E n f e r m a g e m ~ e ~ P r o g r a m a ~ d e ~ P o ́ s-~}$ Graduação em Enfermagem da Universidade Estadual de Maringá, ${ }^{\star * * * \star}$ Enfermeira, docente do Departamento de Enfermagem e Programa de Pós-Graduação em Enfermagem da Universidade Estadual de Maringá

Recebido em 24 de janeiro de 2018; aceito em 30 de março de 2019.

Endereço para correspondência: lara Sescon Nogueira, Rua Hélio Jarreta, 54 Vila Bosque, 87005-030 Maringá PR, E-mail: iara_nogueira@hotmail.com; Rayane Nascimbeni Maldonado: rayane_nascimbeni@hotmail.com; Célia Maria Gomes Labegalini: celia-labegalini@hotmail.com; André Estevam Jaques: andre.jaques@hotmail.com; Ligia Carreira: ligiacarreira@hotmail.com; Vanessa Denardi Antoniassi Baldissera: vanessadenardi@hotmail.com

\section{Resumo}

Introdução: A integração ensino-serviço-comunidade pode transformar as práticas profissionais e a própria organização do trabalho, aprimorando a formação acadêmica e melhorando a qualidade de vida da população. Objetivo: Analisar as contribuições da integração ensinoserviço-comunidade para o processo de formação de acadêmicos de enfermagem e reorganização de práticas na saúde do idoso. Métodos: Estudo qualitativo e descritivo, realizado no período de março a abril de 2015 em um município do estado do Paraná, com nove integrantes de um projeto de extensão universitária em enfermagem. Os dados foram obtidos por meio da aplicação de um questionário semiestruturado utilizando a ferramenta Google Forms ${ }^{\circledR}$, submetidos à Análise de Conteúdo de Bardin. Utilizou-se como referencial teórico-analítico a Educação Permanente em Saúde. Resultados: Emergiram três categorias temáticas: "A integração ensino-serviço-comunidade qualifica os futuros profissionais de saúde", "A integração ensino-serviço-comunidade qualifica o cuidado em saúde" e "A integração ensino-serviçocomunidade possibilita associação de conhecimentos teóricos e práticos". Conclusão: Compreendeu-se a importância da integração ensino-serviço-comunidade para a formação acadêmica e profissional, transformando as práticas na saúde do idoso.

Palavras-chave: saúde do idoso, educação superior, serviços de saúde, educação em enfermagem, serviços de integração docente-assistencial.

\section{Abstract \\ Contributions of the teaching-service-community integration for the formation and transformation of practices in the health of elderly}

Introduction: The teaching-service-community integration can transform professional practices and the organization of work itself, improving academic training and improving the quality of life of the population. Objective: To analyze the contributions of the teaching-service-community integration to the process of training of nursing students and reorganization of practices in the health of the elderly. Methods: A qualitative and descriptive study, carried out from March to April 2015 in a municipality in the state of Paraná, with nine members of a university extension project in nursing. The data were obtained through the application of a semi-structured questionnaire using the Google Forms ${ }^{\circledR}$ tool and submitted to the Bardin Content Analysis. Results: Three 
themes emerged: "Teaching-service-community integration qualifies future health professionals", "The teaching-service-community integration qualifies health care "And" Teaching-servicecommunity integration enables the association of theoretical and practical knowledge ". Conclusion: It was understood the importance of teaching-service-community integration for academic and professional education, transforming practices into the health of the elderly.

Key-words: elderly health, education, higher, health services, education, nursing, teaching care integration services.

\section{Resumen \\ Contribuciones de la integración enseñanza-servicio-comunidad para la formación y transformación de prácticas en la salud de la persona mayor}

Introducción: La integración enseñanza-servicio-comunidad puede transformar las prácticas profesionales y la propia organización del trabajo, mejorando la formación académica y mejorando la calidad de vida de la población. Objetivo: Analizar las contribuciones de la integración enseñanza-servicio-comunidad para el proceso de formación de académicos de enfermería y reorganización de prácticas en la salud de la persona mayor. Métodos: Estudio cualitativo y descriptivo, realizado en el período de marzo a abril de 2015 en un municipio del estado de Paraná, con nueve integrantes de un proyecto de extensión universitaria en enfermería. Los datos se obtuvieron mediante la aplicación de un cuestionario semiestructurado utilizando la herramienta Google Forms $\AA$, sometidos al Análisis de Contenidos de Bardin. Resultados: Emergieron tres categorías temáticas: "La integración educativa-servicio-comunidad califica a los futuros profesionales de la salud", "La integración enseñanza-servicio-comunidad califica el cuidado en salud" y "La integración enseñanza-servicio-comunidad posibilita asociación de conocimientos teóricos y prácticos". Conclusión: Se comprendió la importancia de la integración enseñanza-servicio-comunidad para la formación académica y profesional, transformando las prácticas en la salud del adulto mayor.

Palabras-clave: salud del anciano, educación superior, servicios de salud, educación en enfermería, servicios de integración docente asistencial.

O distanciamento entre o ensino e a realidade dos serviços de saúde é apontado como uma das explicações para a crise do setor saúde no Brasil [1] e também foco de constante indagação a respeito da urgente necessidade de mudança na formação dos profissionais para que se atendam às necessidades de saúde da população [2].

O Sistema Único de Saúde (SUS) deve ordenar a formação de recursos humanos, incrementar a área de atuação dos profissionais e promover o desenvolvimento científico, tecnológico e a inovação, por meio da gestão em saúde que também deve definir os serviços públicos que integram o SUS como campo de prática para o ensino e para a pesquisa com o intuito de melhorar a qualidade da assistência prestada [3-4].

Dessa forma, diversas políticas públicas foram implantadas pelo Ministério da Saúde com a finalidade de melhorar a formação dos profissionais e integrar o ensino aos serviços de saúde em todas as complexidades do SUS, sobretudo na Atenção Primária à Saúde (APS) [5]. Estas trouxeram avanços relacionados à formação dos recursos humanos e a integração entre ensino, serviço e comunidade, porém ainda distante do esperado e necessário [1,5].

De tal modo, a fim de romper com essa lacuna, ações que promovem a integração ensino-serviço-comunidade são necessárias, pois essa parceria pautada no trabalho coletivo, pactuado e integrado de estudantes e docentes dos cursos de formação na área da saúde com trabalhadores que compõem as equipes dos serviços de saúde, junto aos usuários dos serviços, pode levar a transformação das práticas profissionais e da própria organização do trabalho, aprimorando a formação acadêmica [6].

A articulação ensino-serviço-comunidade se dá permeada pela Educação Permanente em Saúde (EPS), em que os processos de educação ocorrem na problematização do processo de trabalho, unindo as necessidades de formação e desenvolvimento dos trabalhadores, contribuindo para uma aprendizagem significativa e na possibilidade de transformação das práticas, melhorando a saúde e a qualidade de vida da população [7]. Nesse sentido, a integração se torna um cenário real de compartilhamento de vivências entre discentes, docentes, profissionais, gestores e comunidade [8-9].

Considerando esse contexto e demanda, um projeto de extensão universitária em enfermagem na atenção ao idoso foi idealizado e vem sendo desenvolvido na perspectiva da 
integração ensino-serviço-comunidade. Resta saber, entretanto, em qual medida essa parceria produz saberes e novos fazeres. Nesse sentido, a presente pesquisa objetivou analisar as contribuições da integração ensino-serviço-comunidade para o processo de formação de acadêmicos de enfermagem e reorganização de práticas na saúde do idoso.

Material e métodos

Esta pesquisa caracterizou-se como estudo descritivo de abordagem qualitativa, realizado no período de março a abril de 2015, tendo como público-alvo 20 integrantes do projeto de extensão universitária intitulado: "Assistência domiciliar de Enfermagem às famílias de idosos dependentes de cuidado (ADEFI)" vinculado a uma universidade pública de ensino, localizada em um município do Norte Central do Estado do Paraná.

O ADEFI vem sendo realizado desde agosto de 2014, por meio de integração ensinoserviço-comunidade, em uma Unidade Básica de Saúde (UBS) localizada no mesmo município em questão, cujo foco é ofertar assistência domiciliar de enfermagem a 116 idosos residentes em uma área que não possui equipe de referência da Estratégia Saúde da Família (ESF). Para tal, os idosos foram caracterizados e estratificados por meio de aplicação de instrumentos específicos e a partir disso foram aplicadas estratégias de enfermagem, como visitas domiciliares, consultas de enfermagem, atividades educativas e grupos operativos.

Após a participação efetiva dos integrantes do projeto ao longo dos meses, os mesmos foram questionados a respeito do que pensam sobre o fato de desenvolverem ações em conjunto com o serviço de saúde/comunidade.

Os critérios de inclusão para participação na pesquisa foram: estar participando efetivamente do projeto em questão e pactuar sua participação livre e autônoma. Não houve critérios de exclusão.

Dessa forma, foram convidados para participar do estudo todos os 20 integrantes do projeto, entre acadêmicos, profissionais de saúde e docentes, por meio de contato pelo endereço eletrônico, para responderem um questionário. Atendendo aos critérios de inclusão, participaram do estudo nove integrantes, sendo oito vinculados ao ensino e um ao serviço. Os outros 11 integrantes do projeto não concordaram em participar do estudo e/ou não responderam o questionário.

O levantamento de dados se deu por meio da aplicação de um questionário semiestruturado, desenvolvido pelos pesquisadores e disponibilizado em ambiente virtual, utilizando a ferramenta Google Forms ${ }^{\circledR}$, ficando as respostas arquivadas nessa mesma ferramenta e disponível aos docentes pesquisadores.

O instrumento foi composto por 13 questões, sendo 10 discursivas e três objetivas, almejando identificar a percepção dos participantes sobre a integração ensino-serviçocomunidade e suas possíveis contribuições para o processo de formação e reorganização de práticas em saúde, além de questões para caracterização dos sujeitos da pesquisa (ocupação, sexo e idade), respectivamente.

Os dados das questões discursivas foram submetidos à Análise de Conteúdo do tipo temática, referindo-se a unidade de análise às contribuições da integração ensino-serviçocomunidade para a formação de acadêmicos de enfermagem e reorganização das práticas na saúde do idoso. A mesma seguiu as três etapas do método: na primeira, denominada de préanálise, foi realizada a organização das respostas na ferramenta virtual e a leitura exaustiva das mesmas, seguindo para a etapa de exploração do material. Nesse momento, as respostas semelhantes foram aglutinadas e codificadas, seguindo para a última etapa, na qual as précategorias dos resultados foram interpretadas e sistematicamente categorizadas compondo as categorias temáticas, de acordo com os significados atribuídos, exaustividade, homogeneidade, exclusividade e fidedignidade dos achados [10].

As questões objetivas voltadas para a caracterização dos participantes, devido a seu caráter complementar, foram analisadas por estatística descritiva simples, por meio de soma de números absolutos e cálculo de média, organizadas na própria ferramenta virtual.

Este estudo teve como referencial teórico-analítico a EPS [7], visto que não é possível referir-se à interface entre ensino, serviço e comunidade sem remeter-se à EPS, já que a mesma possibilita o desenvolvimento dos trabalhadores da área da saúde e também das instituições, ao promover a transformação por meio da análise crítica de problemas, de acordo com seu contexto, reforçando a relação das ações de formação com a gestão dos serviços [7].

$\mathrm{O}$ anonimato das respostas foi garantido, bem como todos os demais preceitos éticos orientados pela Resolução n466/2012 do Conselho Nacional de Saúde [11]. As respostas dos 
participantes foram identificadas com a letra $P$, referindo-se ao termo "participante", seguidas de números arábicos correspondente à ordem de resposta. A pesquisa fazia parte de um estudo mais abrangente cujo projeto foi submetido à apreciação ética pelo Comitê Permanente de Ética em Pesquisa com Seres Humanos da Universidade Estadual do município referido, obtendo parecer favorável no 875.081/2014.

\section{Resultados e discussão}

Compuseram o estudo quatro acadêmicas de graduação do Curso de Enfermagem, quatro alunas do Programa de Pós-Graduação em Enfermagem e uma docente. Todos os nove participantes eram do sexo feminino com idades variando de 19 a 54 anos, média de 27,6 anos.

A partir da análise dos dados emergiram as seguintes categorias temáticas: "A integração ensino-serviço-comunidade qualifica os futuros profissionais de saúde", "A integração ensino-serviço-comunidade qualifica o cuidado em saúde" e "A integração ensino-serviçocomunidade possibilita associação de conhecimentos teóricos e práticos", as quais estão apresentadas a seguir:

\section{A integração ensino-serviço-comunidade qualifica os futuros profissionais de saúde}

Os integrantes evidenciaram a qualificação profissional como resultado da integração ensino-serviço-comunidade, a partir do projeto de extensão, transformando suas práticas assistenciais e formando futuros profissionais de saúde, mais preparados e engajados para atuarem na APS e na atenção ao idoso, conforme as falas a seguir:

"Foi possível vivenciar situações em que a graduação em si não proporciona, nos preparando assim para uma melhor formação profissional". (P1)

"A integração é fundamental para formar profissionais mais comprometidos e preparados para atuar na rede de atenção ao idoso". (P4)

"Percebo que os enfermeiros, principalmente, começaram a aplicar novos conceitos na sua prática e compreender que o planejamento é fundamental para o serviço a nível local. Também tiveram que se apropriar de conhecimentos relacionados às necessidades da pessoa idosa". (P3)

"Acredito que a união da academia, gestão municipal e UBS é muito enriquecedora para a formação de profissionais que atuarão, posteriormente, nesse serviço". (P8)

A integração ensino-serviço-comunidade tem impacto positivo na formação acadêmica, pois ela impulsiona a aprendizagem autêntica e faz com que o aluno desenvolva autonomia $e$ atue com interdisciplinaridade [6,12], além de possibilitar a vivência de fatos apresentados durante as aulas teóricas da graduação, proporcionando contato com os usuários, suas famílias e uma equipe de saúde multiprofissional.

A inserção do acadêmico no serviço de saúde permite que o mesmo vivencie a prática profissional [13], desvelando experiências reais que até então eram conhecidas apenas por conteúdos teóricos dentro das salas de aula, oportunizando aprendizados fundamentais e fortalecendo o processo de ensino-aprendizagem do aluno, como evidenciou-se no presente estudo.

Dessa forma, pode-se inferir que a parceria entre ensino, serviço e comunidade promove a transformação das práticas tradicionais de ensino, as quais são normalmente diretivas, coercivas e acríticas. A partir desta integração, as práticas tradicionais tornam-se participativas e reflexivas, favorecendo a formação de sujeitos capazes de identificar, planejar e intervir sobre os reais problemas de saúde. Outros estudos pontuam que as atividades desenvolvidas no serviço de saúde público pelos acadêmicos de enfermagem permitem a formação de um profissional crítico, competente e humanizado $[9,12,14,19]$, o que também foi evidenciado em nossos resultados.

Diversas atividades do projeto foram realizadas baseando-se na teoria da problematização para o processo de ensino-aprendizagem. Foi adotado como referencial os princípios propostos por Paulo Freire, cuja ação de problematizar acontece a partir da realidade que cerca o sujeito [15]. Tais fundamentos privilegiaram a troca de experiências, saberes e conhecimentos entre os envolvidos (acadêmicos, docentes e profissionais do serviço de saúde), 
considerando o indivíduo com sua história, saberes e vivências individuais e coletivas em um contexto social compartilhado. Neste sentido, todos os envolvidos são detentores de um saber e no instante que a relação dialógica e educativa é estabelecida, os conhecimentos tornam-se convergentes, buscando a mudança individual e coletiva, resultando na transformação da realidade de forma criativa e crítica [15].

A proposta da EPS é coerente com os propósitos da integração ensino-serviçocomunidade ao refletir sobre a formação e o desenvolvimento profissional, os quais envolvem diversos saberes e ocorrem no cotidiano das pessoas e das organizações, resultando no desenvolvimento da capacidade de aprender e de ensinar de todos os atores envolvidos, determinando a qualificação profissional, por meio da efetividade da formação dos estudantes e também da educação permanente dos profissionais [7,12].

\title{
A integração ensino-serviço-comunidade qualifica o cuidado em saúde
}

No que se refere à integração ensino-serviço-comunidade, os participantes relataram que, por meio da integração, houve o aprimoramento do cuidado em saúde, ao auxiliar o planejamento das ações de saúde e consequentemente produzir serviço de qualidade, melhorando o cuidado prestado e a saúde da população idosa, influenciando inclusive, no plano local de saúde do município:

\footnotetext{
"Com os resultados obtidos ficou mais fácil para a unidade criar ações que englobam essa população e assim, fornecer um serviço de qualidade para a comunidade". (P2)
}

\begin{abstract}
"Acredito que compreender como funciona a dinâmica dos serviços de saúde e suas demandas é muito importante para nós da academia, pois assim não produzimos conhecimento apenas por produzir, produzimos para fazer alguma diferença para a população". (P6)
\end{abstract}

"Além de aproximar os alunos da realidade vivenciada pelos profissionais de saúde, ajuda o serviço planejar melhor as ações de saúde, melhorando seu atendimento". (P7)

"Acredito que os dados estão influenciando no planejamento local de saúde". (P8)

Destaca-se que a experiência acadêmica nos serviços de saúde deve ser utilizada para aprendizagem e também para a reflexão sobre a produção de cuidados, o que reforça a importância desta parceria pelo fato de contribuir para o ensino e, consequentemente, melhorar a qualidade do atendimento de saúde qualificando a APS [6]. Assim, os participantes tiveram a oportunidade de protagonizar o processo de implementação das práticas de saúde no que envolve a atenção ao idoso.

A participação dos integrantes do projeto de extensão no planejamento do serviço possibilitou a academia exercer o seu papel social de agente propulsor de mudanças, efetivando também o seu compromisso com a comunidade de tentar transformar o ensino em uma prática justa e democrática, estabelecendo pactos de responsabilização e participação coletiva, engajadas com a necessidade da população [9].

Nesse sentido, a integração ensino-serviço-comunidade contribuiu para o atendimento em saúde prestado à população idosa, ao promover a troca de informações entre discentes e profissionais de saúde, colaborando para a melhoria da assistência. Por um lado, o ensino detém uma visão crítica da literatura e dos procedimentos, e de outro, o serviço possui a visão ampliada da assistência [9], o que contribui para o planejamento das ações de saúde e, consequentemente, redefine as práticas de saúde aos idosos.

Além disso, o acadêmico se torna capaz de identificar as necessidades da comunidade, propor ações em saúde voltadas para estas necessidades previamente identificadas, considerando as possibilidades do serviço de saúde $[6,12]$. Desenvolve sua capacidade de identificação de problemas de saúde existentes e de planejamento de ações em saúde condizentes com as reais possibilidades do serviço, tornando esta parceria benéfica tanto para o aluno, o qual precisa desenvolver suas capacidades com as condições reais do ambiente de trabalho [13] quanto para o serviço, que desenvolve ações voltadas às necessidades de sua comunidade, contribuindo para a melhoria do cuidado e atendimento prestado.

Apesar dos avanços na relação ensino-serviço-comunidade, é comum entre os acadêmicos e docentes uma postura de desconexão com o serviço de saúde, onde as atividades práticas são realizadas [16], o que prejudica o ensino-aprendizagem e a assistência em saúde. Isto ocorre pelo fato da UBS, enquanto serviço da APS, não ser observada com interconexão 
necessária ao processo ensino-aprendizagem e por conta do profissional do serviço, o qual muitas vezes é resistente ou se nega a participar do processo de formação dos acadêmicos [16].

Neste sentido, em nosso estudo, a colaboração e participação dos acadêmicos, docentes e profissionais foram fatores importantes e imprescindíveis para o sucesso das atividades, as quais resultaram em integração ensino-serviço-comunidade, contribuindo de forma significativa para a formação acadêmica dos participantes deste projeto e, principalmente, para a melhoria da qualidade da assistência em saúde prestada a população idosa.

O distanciamento por parte dos profissionais foi notado apenas em relação à participação na pesquisa. Porém, nas atividades desenvolvidas pelo projeto, foram receptivos, participativos e colaboradores, facilitando o diagnóstico das necessidades da população, planejamento, execução e implantação das diversas atividades que, consequentemente, impactaram positivamente no aprimoramento do cuidado em saúde.

No que diz respeito à EPS, permitiu a desfragmentação e integralidade do cuidado, a busca de soluções criativas para os problemas encontrados, o desenvolvimento do trabalho em equipe, a melhoria permanente da qualidade do cuidado à saúde e a humanização do atendimento [7]. Além disso, permitiu a atualização e/ou conhecimento técnico-científico dos profissionais e dos participantes do projeto em um movimento em que todos se beneficiam (estudante, profissional e comunidade) $[8,12,17]$.

A EPS transforma as práticas e os profissionais, pois estes passam a desenvolver 0 conhecimento com base na resolução dos problemas do cotidiano e estabelecem compromisso social e profissional com metas de saúde [7]. Neste sentido, ocorre a desfragmentação das práticas em saúde e reafirma uma assistência humanista baseada na integralidade do cuidado [18], reestruturando as práticas e os serviços de saúde, necessários para a transformação da formação dos profissionais de saúde.

Assim, a aproximação efetiva entre ensino e serviço de saúde (discentes, docentes e profissionais da assistência à saúde) possibilitou a articulação entre o saber e o fazer [8], permitindo o aprendizado e a melhoria do serviço de saúde, tendo em vista que esta parceria permite a prestação de uma assistência à saúde diferenciada, na qual há presença de professores supervisionando os alunos e o atendimento é baseado nas reais necessidades da população, as quais são definidas a partir da realização de pesquisas [9].

\section{A integração ensino-serviço-comunidade possibilita associação de conhecimentos teóricos e práticos}

Segundo os participantes, a integração ensino-serviço-comunidade permitiu a associação de conhecimentos teórico e práticos na atenção ao idoso e possibilitou ao participante aplicar o conhecimento adquirido durante sua formação, tornando indissociável a teoria da prática: "Vivências entre acadêmicos, trabalhadores e população agregam valores que livros e conceitos
de sala de aula não alcançam". (P2) "Aliar a teoria e a prática ajuda muito na formação dos novos profissionais, além disso, a teoria
tem mais valia quando colocada em prática". (P3)

"Um projeto desenvolvido em conjunto possibilita ao acadêmico aplicar a teoria em prática de forma mais realista, tendo em vista que se verifica as necessidades reais da população". (P4)

"Utilizar escalas, índices e ferramentas de gestão permitiu visualizar a implementação de conhecimentos teóricos e sua aplicabilidade na prática". (P5)

"Você percebe que toda a bagagem teórica que você tem e teve até o momento, de nada valeria se não tivesse a prática, e vice-versa. Na verdade, um complementa o outro". (P8)

As asserções acima destacadas tornaram possível analisar que os integrantes do projeto de extensão universitária fazem uso de critérios teóricos para dar suporte aos cuidados de enfermagem prestados aos idosos, mas que no entanto, sua aplicabilidade na prática permitiu validar o conhecimento adquirido dentro de salas de aula.

Os participantes revelaram que a integração ensino-serviço-comunidade auxilia no processo de formação em enfermagem, pois associa o aprendizado teórico à atividade prática, ressaltando a rotina da UBS, considerando a sua realidade e princípios, e permitindo promover 
uma assistência baseada na realidade vivenciada (tanto serviço ofertado quanto as necessidades dos usuários), alcançando com isso, melhores resultados nas intervenções realizadas com os idosos e adquirindo experiências em situações nas quais o aluno irá futuramente exercer seu papel como profissional.

A integração entre ensino junto ao serviço tem sido objeto de estudo em diversos estudos $[2,6,8-9,13-14,16,19]$, sendo considerado um dispositivo desencadeador de mudanças, mediante a aproximação entre aprendizagem e prática profissional [6]. A inserção de acadêmicos e docentes nos serviços fortalecem os conhecimentos norteadores das políticas de saúde e EPS, propiciam a realização de um maior número de atividades educativas e discussões entre docentes e trabalhadores, além de envolver a participação dos profissionais do serviço no planejamento e execução das estratégias pedagógicas na prática [19]. Dessa forma, a integração ensino-serviço-comunidade contribui para estreitar a distância entre teoria e prática, intensificando a atuação dos envolvidos como agentes de mudanças e redefinindo as práticas em saúde.

A troca de informações entre ensino e serviço proporcionada pela EPS, permite ao acadêmico a desfragmentação de sua formação, possibilitando a aplicação dos conteúdos teóricos na prática, representando o encontro entre a saúde e educação. Nesta perspectiva, o processo de trabalho é o eixo central da aprendizagem e por meio da utilização de metodologias ativas de aprendizagem é possível realizar a problematização da realidade [20]. Neste sentido, o acadêmico pode aplicar seu conhecimento técnico-científico na prática por meio da problematização, além disso, é estimulado aprimorar e a adaptar seu conhecimento técnicocientífico à prática em saúde baseada em cada indivíduo e sua respectiva realidade.

Sobre as exigências do Ministério da Educação para o funcionamento de cursos de enfermagem no que diz respeito à integração ensino-serviço-comunidade, ainda se busca uma reformulação e maior inserção nos currículos acadêmicos, sendo disponíveis atividades promotoras de integração durante a formação acadêmica, como o estágio curricular supervisionado, o Programa de Educação pelo Trabalho para Saúde (PET-Saúde), o Programa Nacional de Reorientação da Formação Profissional em Saúde (Pró-Saúde), além de projetos de extensão [21], como é o caso da presente pesquisa.

Destaca-se como limitações do estudo a ausência da participação dos profissionais do serviço que, indiscutivelmente, acrescentaria novas perspectivas e olhares para a integração ensino-serviço-comunidade. Sugere-se a realização de novos estudos que abordem a temática em questão e que desvelem a perspectiva do serviço e também da comunidade, também implicados na integração ensino-serviço-comunidade.

Os participantes relataram a importância da integração ensino-serviço-comunidade para a formação acadêmica e profissional, sendo apontada como colaboradora no processo de qualificação do cuidado em saúde e na consequente transformação das práticas de saúde na atenção ao idoso.

Observamos que a parceria ensino-serviço-comunidade permitiu, por meio da pesquisa, a identificação das necessidades de saúde da população idosa, contribuindo para a elaboração de ações em saúde voltadas para as reais necessidades, considerando as possibilidades do serviço, associando teoria à prática.

Portanto, integração ensino-serviço-comunidade foi considerada um meio de aprendizagem tanto para acadêmicos quanto para os profissionais, concretizando a EPS, na medida em que contribuiu para a melhoria da qualidade da assistência balizada pelas trocas de saberes e fazeres em saúde.

1. Ministério da Saúde (BR). Secretaria de Gestão do Trabalho e da Educação em Saúde. Departamento de Gestão da Educação em Saúde. Programa Nacional de Reorientação da Formação Profissional em Saúde. Brasília: Ministério da Saúde, 2005.

2. Mira QLM, Barreto RMA, Vasconcelos MIO. Impacto do Pet-Saúde na formação profissional: uma Revisão Integrativa. Rev Baiana Saúde Pública 2016;40(2):514-31. https://doi.org/10.22278/2318-2660.2016.v40.n2.a1682 
3. Mattia BJ, Kleba ME, Prado ML. Nursing training and professional practice: an integrative review of literature. Rev Bras Enferm 2018;71(4):2039-49. https://doi.org/10.1590/0034-7167-2016-0504

4. Brasil. Lei oํ 8080, de 19 de setembro de 1990. Dispõe sobre as condições para a promoção, proteção e recuperação da saúde, a organização e o funcionamento dos serviços correspondentes e dá outras providências. Diário Oficial da União. Brasília, 20 set. 1990; Seção 1.

5. Vasconcelos ACF, Stedefeldt E, Frutuoso MFP. Uma experiência de integração ensinoserviço e a mudança de práticas profissionais: com a palavra, os profissionais de saúde. Interface Comun Saúde Educ 2016;20(56):147-58. https://doi.org/10.1590/1807$\underline{57622015.0395}$

6. Vendruscolo C, Prado ML; Kleba ME. Integração ensino-serviço no âmbito do Programa Nacional de Reorientação da Formação Profissional em Saúde. Ciênc Saúde Colet 2016;21(9):2949-60. https://doi.org/10.1590/1413-81232015219.12742015

7. Ministério da Saúde (BR). Secretaria de Gestão do Trabalho e da Educação na Saúde. Departamento de Gestão da Educação em Saúde. Política Nacional de Educação Permanente em Saúde. Brasília: Ministério da Saúde; 2009. 64p.

8. Brehmer LCF, Ramos FRS. Experiências de integração ensino-serviço no processo de formação profissional em saúde: revisão integrativa. Rev Eletrônica Enferm 2014;16(1):228-237. https://doi.org/10.5216/ree.v16i1.20132

9. Silva FA, Costa NMSC, Lampert JB, Alves R. Papel docente no fortalecimento das políticas de integração ensino-serviço-comunidade: contexto das escolas médicas brasileiras. Interface Comun Saúde Educ 2018;22(suppl1):1411-23. https://doi.org/10.1590/1807-57622017.0062

10. Bardin L. Análise de conteúdo. Lisboa: Edições 70; 2011.

11. Ministério da Saúde (BR). Conselho Nacional de Saúde. Diretrizes e normas regulamentadoras de pesquisa envolvendo seres humanos. Resolução n. 466, de 12 de dezembro de 2012. Ministério da Saúde: Brasília; 2012.

12. Vendruscolo C, Ferraz F, Prado ML, Kleba ME, Reibnitz KS. Integração ensino-serviço e sua interface no contexto da reorientação da formação na saúde. Interface Comun Saúde Educ 2016;20(59):1015-25. https://doi.org/10.1590/1807-57622015.0768

13. Kloh D, Reibnitz KS, Corrêa AB, Lima MMD, Cunha APD. Integração ensino-serviço no contexto do projeto político-pedagógico de cursos de enfermagem. Rev Enferm UFPE on line 2017;11(supl.11):4554-62. https://periodicos.ufpe.br/revistas/revistaenfermagem/article/view/231194/25185

14. Corrêa AB, Reibnitz KS, Kloh D, Prado ML, Rodrigues J, Lima MM. Contribuições do programa pró-saúde: uma visão dos egressos de enfermagem. Rev Enferm UFPE on line 2017;11(2):567-75. https://periodicos.ufpe.br/revistas/revistaenfermagem/article/view/11975/14524

15. Freire P. Pedagogia do Oprimido. 65 ed. Rio de Janeiro/São Paulo: Paz \& Terra; 2018. 256p.

16. Cezario JEP, Daher DV, Nolasco MFS. Programa de Educação pelo Trabalho (PET) em saúde: articulação entre formação e integração ensino-serviço. Rev Enferm UFRJ 2015;23(5):644-8. https://doi.org/10.12957/reueri.2015.7979

17. Schott M. Articulação ensino-serviço: estratégia para formação e educação permanente em saúde. Revista Família, Ciclos de Vida e Saúde no Contexto Social 2018;6(2):2648. Disponível em: http://www.redalyc.org/articulo.oa?id=497955513017

18. Teixeira RC, Corrêa RO, Silva EM. Percepções dos discentes de terapia ocupacional sobre a experiência de integração ensino-serviço-comunidade. Cad Bras Ter Ocup 2018;26(3):617-25. https://doi.org/10.4322/2526-8910.ctoAO1167

19. Faria L, Quaresma MA, Patiño RA, Siqueira R, Lamego G. Integração ensino-serviçocomunidade nos cenários de práticas na formação interdisciplinar em Saúde: uma experiência do Programa de Educação pelo Trabalho para a Saúde (PET-Saúde) no sul da Bahia, Brasil. Interface Comun Saúde Educ 2018;22(67):1257-66. https://doi.org/10.1590/1807-57622017.022

20. Silva DSJR, Duarte LR. Educação permanente em saúde. Rev Fac Cienc Med Sorocaba 2015;17(2):104-5. Disponível em: http://revistas.pucsp.br/index.php/RFCMS/article/viewFile/23470/pdf 
21. Baldoino AS, Veras RM. Análise das atividades de integração ensino-serviço desenvolvidas nos cursos de saúde da Universidade Federal da Bahia. Rev Esc Enferm USP 2016;50(spe):17-24. https://doi.org/10.1590/S0080-623420160000300003 